\title{
An Exactly Two-to-One Map from an Indecomposable Chainable Continuum
}

by

\author{
Jerzy KRZEMPEK
}

Presented by Andrzej LASOTA

Summary. It is shown that a certain indecomposable chainable continuum is the domain of an exactly two-to-one continuous map. This answers a question of Jo W. Heath.

In 1939 O. G. Harrold [4] proved that the interval [0,1] is not the domain of any (exactly) two-to-one map $\left({ }^{1}\right)$. Further spaces do not support such maps: any connected graph with odd Euler characteristic [3], the real line [10], the cube $[0,1]^{n}$ for any finite $n[1]$, the Knaster bucket handle continuum $\left({ }^{2}\right)$ [10], other Knaster type continua and some of solenoids [2]. In 1961 J. Mioduszewski [10] asked if there exists a two-to-one map from the pseudo-arc, the only (up to homeomorphism) hereditarily indecomposable $\left(^{3}\right)$ chainable continuum. This question is still open.

The first example of a chainable continuum that does support a two-toone map was given in [5]. J. W. Heath [5-9] asked if any indecomposable chainable continuum can be the domain of a two-to-one map.

In this note we answer the question of Heath in the affirmative.

A continuum is chainable when for every $\varepsilon>0$, the continuum is covered by open sets $U_{1}, \ldots, U_{n(\varepsilon)}$ of diameters $\leq \varepsilon$ such that $U_{i}$ meets $U_{j}$ iff

2000 Mathematics Subject Classification: Primary 54C10; Secondary 54F15, 54E40.

Key words and phrases: chainable continuum (= arclike continuum), indecomposable continuum, Knaster bucket handle continuum, exactly two-to-one map.

$\left({ }^{1}\right)$ All maps are meant to be continuous, and all spaces are metric. A function is called (exactly) $k$-to-one if each point-inverse of the function has exactly $k$ elements.

$\left(^{2}\right)$ A continuum is a connected compact space.

$\left(^{3}\right)$ A continuum is indecomposable if it is not the union of two proper subcontinua. A continuum is hereditarily indecomposable if each subcontinuum is indecomposable. 
$|i-j| \leq 1$. We say that points $x, y$ in a chainable continuum are opposite end points if for every $\varepsilon>0$, we can assume that $x \in U_{1}$ and $y \in U_{n(\varepsilon)}$.

A chainable continuum need not necessarily have a pair of opposite end points. However, if it does, then it is irreducible between these points $\left({ }^{4}\right)$.

We need the following result from [5, Example 3], attributed to W. Lewis.

EXAMPlE 1. There exist: a (decomposable) chainable continuum $X$ with opposite end points $0,1 \in X$ and a map $f$ from $X$ such that card $f^{-1} f(x)=2$ iff $x \in X \backslash\{0,1\}$. Hence card $f^{-1} f(0)=$ card $f^{-1} f(1)=1$.

We proceed to the construction of our main example:

EXAMPLE 2. There exists an indecomposable chainable continuum that is the domain of an exactly two-to-one map.

Construction. Consider the Cantor ternary set $C \subset \mathbb{R}$. Any element $\sum_{i=1}^{\infty} 2 a_{i} / 3^{i} \in C$, where $a_{i} \in\{0,1\}$, will be denoted by $a_{1} a_{2} \ldots a_{i} \ldots$ It will be convenient to construct the domain and the image of our two-to-one map simultaneously. So, take Lewis's continuum $X$ of Example 1 and its image $f(X)$. We can assume that $f(0)=0, f(1)=1$, and $X \cap f(X)=\{0,1\}$. Then consider the following two products: $D=\{0,1\} \times C \subset[X \cup f(X)] \times C$. Their elements will be denoted by $a=a_{0} . a_{1} a_{2} a_{3} \ldots$, where $a_{0} \in X \cup f(X)$ and $a_{1} a_{2} a_{2} \ldots \in C$. We also put $o=0.000 \ldots$

We shall identify certain pairs of points in $D \backslash\{o\}$, which will resemble the well known construction of the Knaster bucket handle continuum. Consider the switching operation $\widehat{0}=1$ and $\widehat{1}=0$. For any element $a \in D \backslash\{o\}$ let us put $n(a)=\min \left\{i: a_{i}=1\right\}$, and then let us identify

$$
a \sim \underbrace{0.00 \ldots 0}_{n(a) \text { zeros }} 1 \widehat{1 a_{n(a)+1}} \widehat{a_{n(a)+2}} \widehat{a_{n(a)+3}} \widehat{a_{n(a)+4}} \cdots
$$

Consistently, when $a_{0}=1$, we write $1 . a_{1} a_{2} a_{3} \ldots \sim 1 . \widehat{a_{1}} \widehat{a_{2}} \widehat{a_{3}} \ldots$ We have thus obtained an upper semicontinuous equivalence relation $\sim$ on $[X \cup f(X)] \times C$. Denote by $p$ the natural projection that carries $a \in[X \cup f(X)] \times C$ to its equivalence class $[a]_{\sim}$. Let $K, L$, and $E$ denote $p(X \times C), p[f(X) \times C]$, and $p(D)$ respectively. See Figure 1.

The space $K$ will be the domain of the map to be defined. So, let us look at some of its subspaces. Consider all copies $X \times\{c\}$ of $X$, where $c \in C$ are the ends of open intervals contained in $\mathbb{R} \backslash C$. The images $p(X \times\{c\})$ are no longer pairwise disjoint. However, we can arrange them in a sequence $\left\{F^{n}\right\}_{n=1}^{\infty}$ such that

$\left({ }^{4}\right)$ A continuum $C$ is irreducible between points $x, y \in C$ if no proper subcontinuum of $C$ contains both $x$ and $y$. 


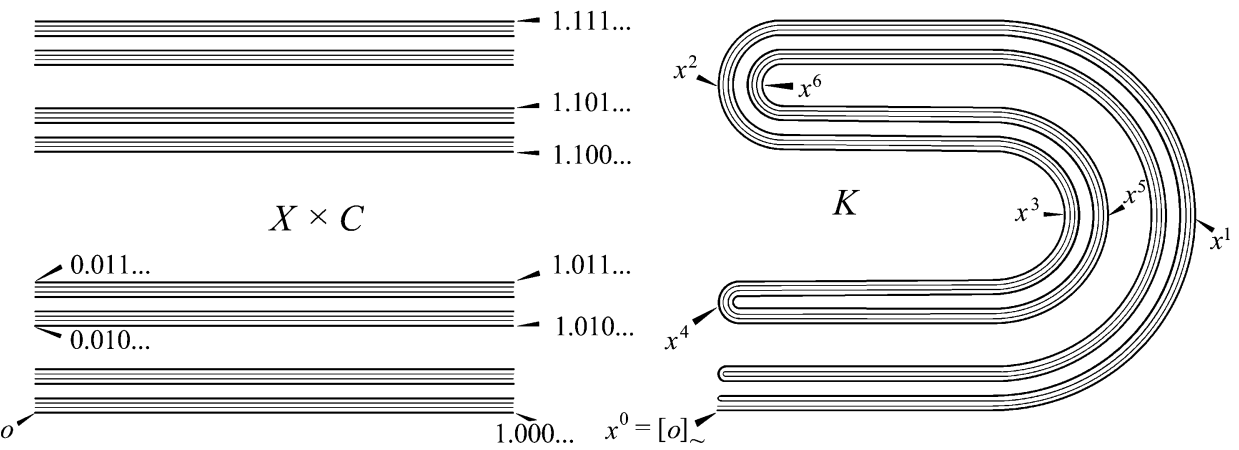

Fig. 1. The construction of the domain $K$ of Example 2. In view of a remark at the end of the paper, the auxiliary continuum $L=g(K)$ has the same structure. Moreover, $[p \mid(f(X) \times C)] \circ\left(f \times \operatorname{id}_{C}\right)=g \circ[p \mid(X \times C)]$.

- $[o]_{\sim} \in F^{1}$,

- $\operatorname{card}\left(F^{n} \cap F^{n+1}\right)=1$ for each $n$,

- $F^{n} \cap F^{m}=\emptyset$ whenever $|n-m|>1$.

$K$ is connected as it has a dense connected subspace, $Y=\bigcup_{n=1}^{\infty} F^{n}$. The chainability of $K$ results from the fact that 0 and 1 are opposite end points of $X$. The indecomposability of $K$ results from this claim: If $M \subset K$ is a subcontinuum that contains $[o]_{\sim}$, then $M \subset Y$ or $M=K$.

To prove the claim, let $x^{0}=[o]_{\sim}$, and denote by $x^{n}$ the only point in $F^{n} \cap F^{n+1}$. There are two possibilities: (1) There is an $x^{n} \notin M$. Then there is an open neighbourhood $V \ni x^{n}$ that does not meet $M$, and $M$ is contained in some component of $K \backslash V$. Since $[o]_{\sim} \in M$, this component is contained in $\left(F^{1} \cup \cdots \cup F^{n}\right) \backslash V$. Hence $M \subset Y$. (2) If each $x^{n}$ is in $M$, then $F^{n} \subset M$ for each $n$. Indeed, chainable continua are hereditarily unicoherent $\left({ }^{5}\right)$ (see $\left[11,12.2\right.$ and 12.11]). Therefore, $F^{n} \cap M$ is a continuum. Moreover, $F^{n}$ is homeomorphic to $X ; x^{n-1}$ and $x^{n}$ are opposite end points of $F^{n}$. Since $F^{n}$ is irreducible between $x^{n-1}$ and $x^{n}$, we have $F^{n} \subset F^{n} \cap M \subset M$. Thus $Y \subset M$, and as $Y$ is dense in $K$, we obtain $K=M$. The proof of the claim is complete.

The following formula defines a continuous function $g: K \stackrel{\text { onto }}{\longrightarrow} L$ :

$$
g\left(\left[a_{0} \cdot a_{1} a_{2} a_{3} \ldots\right]_{\sim}\right)=\left[f\left(a_{0}\right) \cdot a_{1} a_{2} a_{3} \ldots\right]_{\sim} .
$$

Observe that $g \mid E=\operatorname{id}_{E}$, and $L \backslash E$ is the two-to-one image of $K \backslash E$ under $g$. Since $E$ is homeomorphic to the Cantor set, there exists a map $h$ from $L$ such that $h \mid E$ is two-to-one, $h \mid L \backslash E$ is one-to-one, and $h(E) \cap h(L \backslash E)=\emptyset$. Finally, $h \circ g$ is a two-to-one map from $K$ onto $h(L)$.

$\left({ }^{5}\right)$ A continuum is hereditarily unicoherent if any two subcontinua have connected intersection. 
Remarks. Our continuum $K$ and the Knaster bucket handle continuum are constructed according to the same schema. Since different patterns (Lewis' continuum $X$ or an arc, respectively) are used in these two cases, the resulting continua have different properties: there exists no two-to-one map from the Knaster bucket handle continuum ([10], see also [2]).

Lewis' construction [5] allows more than Example 1 says. His continuum $X$ is built of certain continua $C_{i}$; each of them has opposite end points $p_{i}$ and $q_{i}$, and the restrictions $f \mid C_{i}: C_{i} \rightarrow f\left(C_{i}\right)$ are open maps. Looking at the proof of Theorem 1.0 in [12] (the open image of a chainable continuum is chainable), we see that $f\left(p_{i}\right)$ and $f\left(q_{i}\right)$ are opposite end points of $f\left(C_{i}\right)$. Then we infer that $f(0)$ and $f(1)$ in Example 1 are opposite end points of the chainable image $f(X)$. Therefore, we can prove that the continuum $L=g(K)$ in our construction of Example 2 is chainable and indecomposable.

In general the image $h(L)$ need not be indecomposable, even if $L$ is. The author does not know whether the two-to-one image of an indecomposable chainable continuum can be indecomposable.

\section{References}

[1] A. V. Chernavskiǔ, The impossibility of a strictly double continuous partition of a homology cube, Dokl. Akad. Nauk SSSR 144 (1962), 286-289 (in Russian); English transl.: Soviet Math. Dokl. 3 (1962), 726-729.

[2] W. Dębski, Two-to-one maps on solenoids and Knaster continua, Fund. Math. 141 (1992), 277-285.

[3] P. Gilbert, n-to-one mappings of linear graphs, Duke Math. J. 9 (1942), 475-486.

[4] O. G. Harrold, The non-existence of a certain type of continuous transformation, Duke Math. J. 5 (1939), 789-793.

[5] J. W. Heath, Treelike continua and exactly $k$-to-one functions, Proc. Amer. Math. Soc. 105 (1989), 765-772.

[6] -, 2-to-one maps with hereditarily indecomposable images, ibid. 113 (1991), $839-846$.

[7] -, 2-to-1 maps on hereditarily indecomposable continua, Trans. Amer. Math. Soc. 328 (1991), 433-444.

[8] - Four key questions in the theory of 2-to-1 maps, in: Topology. Theory and Applications, II, Colloq. Math. Soc. János Bolyai 55, North-Holland, Amsterdam, 1993, 301-305.

[9] —, Exactly k-to-1 maps: from pathological functions with finitely many discontinuities to well-behaved covering maps, in: Continua. With the Houston Problem Book, Lecture Notes in Pure and Appl. Math. 170, Dekker, New York, 1995, 89-102.

[10] J. Mioduszewski, On two-to-one continuous functions, Rozprawy Mat. (Dissertationes Math.) 24 (1961).

[11] S. B. Nadler, Continuum Theory. An Introduction, Monogr. Textbooks Pure Appl. Math. 158, Dekker, New York, 1992. 
[12] I. Rosenholtz, Open maps of chainable continua, Proc. Amer. Math. Soc. 42 (1974), 258-264.

Jerzy Krzempek

Institute of Mathematics

Technical University of Silesia

Kaszubska 23

44-100 Gliwice, Poland

E-mail: krzem@zeus.polsl.gliwice.pl

Received March 12, 2004;

received in final form August 2, 2004 